ARTICLE

https://doi.org/10.1038/s41467-018-07978-1

\title{
Low-frequency vibrational modes of stable glasses
}

Lijin Wang ${ }^{1,2}$, Andrea Ninarello ${ }^{3,4}$, Pengfei Guan ${ }^{1}$, Ludovic Berthier (10 ${ }^{3}$, Grzegorz Szamel ${ }^{2}$ \& Elijah Flenner ${ }^{2}$

Unusual features of the vibrational density of states $D(\omega)$ of glasses allow one to rationalize their peculiar low-temperature properties. Simulational studies of $D(\omega)$ have been restricted to studying poorly annealed glasses that may not be relevant to experiments. Here we report on $D(\omega)$ of zero-temperature glasses with kinetic stabilities ranging from poorly annealed to ultrastable glasses. For all preparations, the low-frequency part of $D(\omega)$ splits between extended and quasi-localized modes. Extended modes exhibit a boson peak crossing over to Debye behavior $\left(D_{\mathrm{ex}}(\omega) \sim \omega^{2}\right)$ at low-frequency, with a strong correlation between the two regimes. Quasi-localized modes obey $D_{\text {loc }}(\omega) \sim \omega^{4}$, irrespective of the stability. The prefactor of this quartic law decreases with increasing stability, and the corresponding modes become more localized and sparser. Our work is the first numerical observation of quasi-localized modes in a regime relevant to experiments, and it establishes a direct connection between glasses' stability and their soft vibrational modes

\footnotetext{
${ }^{1}$ Beijing Computational Science Research Center, 100193 Beijing, China. ${ }^{2}$ Department of Chemistry, Colorado State University, Fort Collins, CO 80523, USA ${ }^{3}$ Laboratoire Charles Coulomb (L2C), University of Montpellier, CNRS, 34095 Montpellier, France. ${ }^{4}$ CNR-ISC, Uos Sapienza, Piazzale A. Moro 2, 00185 Roma, Italy. Correspondence and requests for materials should be addressed to P.G. (email: pguan@csrc.ac.cn) or to E.F. (email: flennere@gmail.com)
} 
A morphous solids exhibit universal low-temperature properties, seen for instance in the heat capacity and thermal conductivity ${ }^{1}$, that differ remarkably from crystal physics. These properties are related to the vibrational density of states $D(\omega)$. For a continuous elastic medium in three dimensions, low-frequency excitations are phonons, and the density of states follows $D(\omega)=A_{\mathrm{D}} \omega^{2}$, where $A_{\mathrm{D}}$ is given by Debye theory ${ }^{2}$. A well-known universal feature of amorphous solids is an excess in vibrational modes over the Debye prediction that results in a peak in $D(\omega) / \omega^{2}$ at an intermediate frequency, called the boson peak $^{3-6}$.

More recently, another source of 'excess modes' has been identified in computer simulations of model glasses ${ }^{7-12}$. It is composed of quasi-localized low-frequency modes with a density obeying $D_{\text {loc }}(\omega) \sim \omega^{4}$. Quasi-localized modes are observed at frequencies significantly lower than the boson peak and the link between the two phenomena is not immediate, despite some indications that they may be connected ${ }^{8,13}$. The quartic law was predicted long ago using phenomenological models ${ }^{14,15}$, reanalyzed over the years ${ }^{16-18}$, and remains the focus of intense research ${ }^{19,20}$. These predictions differ from two recent mean-field approaches ${ }^{21,22}$, which predict instead a universal non-Debye behavior that is quadratic in all spatial dimensions, also reported numerically ${ }^{23}$. Interest in the low-frequency localized modes extends beyond connections to theoretical models and the boson peak. It was suggested that these modes are correlated with irreversible structural relaxation in the supercooled liquid state ${ }^{24}$, and that the spatial distribution of these soft modes is correlated with rearrangements upon mechanical deformation and plasticity ${ }^{25-28}$. Localized defects are also central to theoretical descriptions of glass properties at cryogenic temperatures ${ }^{29,30}$.

Recent numerical insights were obtained for glasses that are very different from the ones studied experimentally, since they are prepared with protocols operating on timescales that differ from experimental ones by as many as ten orders of magnitude ${ }^{31}$. It is therefore unknown whether any of the vibrational, thermal, or mechanical properties derived from earlier computational study of the density of states is experimentally relevant. For example, it was reported ${ }^{9,32}$ that $D_{\text {loc }}(\omega) \sim \omega^{\beta}$ with $\beta$ ranging from 3 to 4 depending on the glass's stability, with $\beta=4$ for the two most stable simulated glasses created by cooling at a constant rate. It remains unclear, however, whether $\beta$ would be different for glasses with stability comparable to that of the experimental glasses.

Our main achievement is to extend studies of the vibrational density of states of computer glasses to an experimentally relevant regime of glass stability for the first time. To this end, we build on the recent development of a Monte Carlo method that allows us to equilibrate supercooled liquids down to temperatures below the experimental glass transition ${ }^{33-35}$ to prepare glasses that cover an unprecedented range of kinetic stability, from extremely poorly annealed systems to ultrastable glasses. We thus match the large gap between previous numerical findings and the experimental regime ${ }^{36}$. Recent studies have shown that that such stable glasses may differ qualitatively from ordinary computer glasses $35,37,38$. For example, qualitatively different yielding behavior of well-annealed glasses compared to that of poorly annealed glasses was reported in ref. ${ }^{38}$. Since rearrangements upon mechanical deformation are correlated with the spatial distribution of soft modes, this result suggested that the density of states could also evolve dramatically with the stability.

\section{Results}

System preparation. We prepare glasses by instantaneously quenching supercooled liquids equilibrated at parent temperature
$T_{\mathrm{p}}$ to $T=0$, so that $T_{\mathrm{p}}$ uniquely controls the glass stability. We find that the low-frequency part of the vibrational density of states changes considerably when $T_{\mathrm{p}}$ varies, thus offering a direct link between soft vibrational modes and kinetic stability. Following earlier work ${ }^{8,10}$, we divide modes into extended and quasilocalized ones. As found for high parent temperature glasses ${ }^{7-10}$, the density of states of the quasi-localized modes follows $D_{\mathrm{loc}}=$ $A_{4} \omega^{4}$, with the same quartic exponent for all glass stabilities. Our work thus establishes the relevance of earlier findings about quasi-localized modes and their effect on the density of states in the experimentally relevant regime of glass stability. In addition, we find that the overall scale $A_{4}$ decreases surprisingly rapidly when $T_{\mathrm{p}}$ decreases, showing that the density of the quasi-localized modes is highly sensitive to the glass stability. This rapid decrease contrasts with the modest changes found for other structural quantities, such as mechanical moduli, sound speed, and Debye frequency. Quasi-localized modes also become sparser and increasingly localized at low $T_{p}$, and so the identification of soft localized modes as relevant glassy defects controlling the physics of amorphous solids becomes more convincing near the experimental glass transition. Our results also suggest that ultrastable glasses contain significantly fewer localized excitations than ordinary glasses, which appears consistent with recent experiments $^{39-41}$.

We simulate a polydisperse glass forming system in three dimensions, which is a representative glass-forming computer model $^{33}$. We use the swap Monte Carlo algorithm to prepare independent equilibrated configurations at parent temperatures $T_{\mathrm{p}}$ ranging from above the onset temperature of slow dynamics $T_{\mathrm{o}} \approx 0.200$, down to $T_{\mathrm{p}}=0.062$, which is about $60 \%$ of the modecoupling temperature $T_{\mathrm{c}} \approx 0.108 \quad\left(T_{\mathrm{c}}\right.$ marks a crossover to activated dynamics and corresponds typically to the lowest temperature accessed by standard molecular dynamics). Importantly, our lowest $T_{\mathrm{p}}$ is lower than the estimated experimental glass temperature $T_{\mathrm{g}} \approx 0.072^{33}$, and no previous computational study has explored such range of glass stability. In addition, we also use a very high parent temperature which we refer to as $T_{\mathrm{p}}=\infty$. We then probe vibrational properties of zerotemperature glasses produced by an instantaneous quench from equilibrated configurations at different $T_{\mathrm{p}}$. The specific simulation details are provided in Methods.

Macroscopic properties. We begin by presenting macroscopic properties of the glasses as a function of the parent temperature $T_{\mathrm{p}}$. The inherent structure energy $E_{\mathrm{IS}}$ is directly related to the mobility of the particles ${ }^{42}$, and thus we show $E_{\mathrm{IS}}$ in Fig. 1a as an indicator of the increased stability of the glass. $E_{\mathrm{IS}}$ deviates from its high-temperature plateau when $T_{\mathrm{p}}$ becomes smaller than the onset temperature, and decreases further with decreasing $T_{\mathrm{p}}{ }^{43}$. Similarly, the bulk modulus $B$ decreases modestly with decreasing $T_{\mathrm{p}}$ (Fig. 1b). By contrast, the shear modulus $G$ in Fig. 1c remains nearly temperature-independent until themode-coupling temperature, which is below the onset temperature, and then the shear modulus increases with decreasing $T_{\mathrm{p}}$. Associated with the increase in the shear modulus is a decrease in the Debye level $A_{\mathrm{D}}=3 / \omega_{\mathrm{D}}^{3}$, where the Debye frequency $\omega_{\mathrm{D}}=\left[\left(18 \pi^{2} \rho\right) /\left(c_{1}^{-3}+2 c_{\mathrm{t}}^{-3}\right]^{1 / 3}\right.$. The decrease of $A_{\mathrm{D}}$ is mainly controlled by the increase of the shear modulus since the transverse speed of sound $c_{\mathrm{t}}=\sqrt{G / \rho}$ is 2.4-2.6 times smaller than the longitudinal speed of sound $c_{1}$. The overall relative variations of mechanical moduli and Debye frequency are, however, relatively mild given the broad range of glass stabilities covered in Fig. 1.

Classification of quasi-localized and extended modes. By examining the participation ratio $P(\omega)$ as a function of $\omega$ at 
a

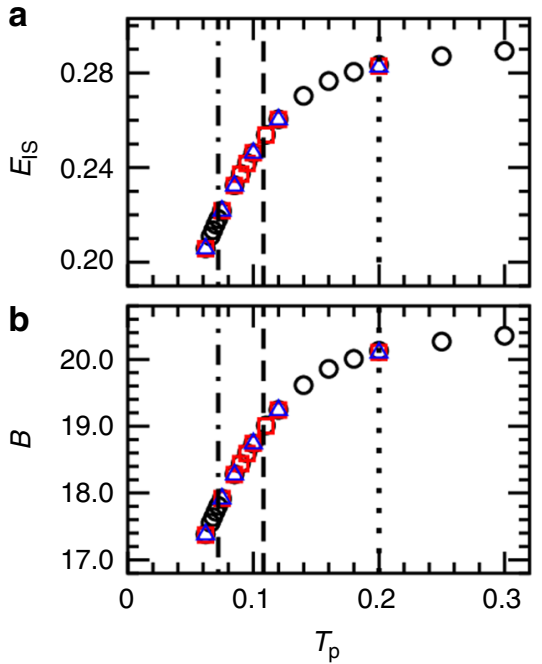

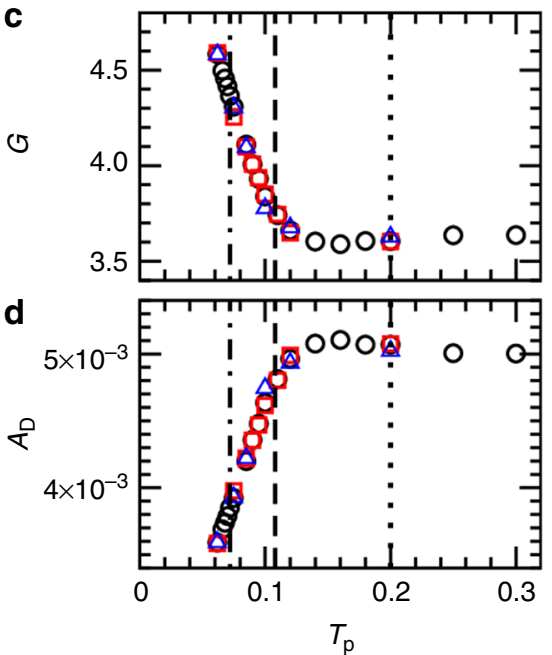

Fig. 1 Macroscopic properties. a Inherent structure energy $E_{I S}$; b Bulk modulus $B$; c Shear modulus $G$; d Debye level $A_{D}$. In all panels, the vertical dasheddotted, dashed, and dotted lines mark the positions of $T_{\mathrm{g},} T_{\mathrm{c}}$, and $T_{\mathrm{o}}$, respectively. Data shown are for $N=48,000$ (circles), 96,000 (squares), and 192,000 (triangles)

different parent temperatures (see Fig. 2), we observe all the features that characterize the $T_{\mathrm{p}}$-dependence of the density of states. A value of $P(\omega)=1$ indicates a mode where all the particles participate equally, a value of $P(\omega)=N^{-1}$ indicates a mode where only one particle participates, and a value of $P(\omega)=2 / 3$ indicates a plane wave. The sharp peaks in $P(\omega)$ at low frequencies are due to the phonon modes, with the first peak corresponding to the first allowed transverse phonon at $\omega_{\mathrm{t}}=c_{\mathrm{t}} 2 \pi / L, L$ being the box length. An increase in $\omega_{\mathrm{t}}$ indicates an increase in $c_{\mathrm{t}}=\sqrt{G / \rho}$. The low-frequency modes can be naturally divided into quasilocalized modes (small $P$ ) and extended modes (large $P$ ) through an appropriate thresholding procedure ${ }^{8,10}$, this decomposition becoming sharper as $L$ increases and $T_{\mathrm{p}}$ decreases. The value $P_{0}=0.006$ is appropriate, as shown in Fig. 2, but we checked that our results are not qualitatively affected by a reasonable change of $P_{0}$. As $T_{\mathrm{p}}$ decreases, phonon modes shift to larger frequencies, as expected from the evolution of the mechanical moduli, whereas quasi-localized modes become increasingly localized and well-separated from the phonons. We also checked that our results hold for small system sizes where allowed phonon modes are shifted to much higher frequencies ${ }^{7}$.

Properties of quasi-localized modes. We examined the density of states for the quasi-localized modes $D_{\text {loc }}(\omega)$, which are shown in Fig. 3a for a few representative $T_{\mathrm{p}}$. At low frequencies, $D_{\text {loc }}(\omega)$ $=A_{4} \omega^{4}$ for each parent temperature with a prefactor $A_{4}$ that depends on the glass stability. We show the resulting $A_{4}\left(T_{\mathrm{p}}\right)$ in Fig. 3b. The prefactor $A_{4}$ stays nearly constant for high enough $T_{\mathrm{p}}$, but decreases sharply when $T_{\mathrm{p}}$ decreases below the modecoupling temperature $T_{\mathrm{c}}$. This observation is robust against changing the system size. The decrease of $A_{4}$ at low $T_{\mathrm{p}}$ correlates well with the evolution of shear modulus and Debye level in Fig. 3c, d. We note that a study of less stable glasses ${ }^{32}$ found an increase in the lowest frequency of quasi-localized modes with decreasing parent temperature, which, under certain assumptions, may be related to the change of $A_{4}$ reported here. A major result of our study is that the quartic law governing $D_{\mathrm{loc}}(\omega)$ is obeyed irrespective of the glass stability, thus extending the validity of previous findings to the experimentally relevant regime.

In Fig. $3 \mathrm{c}$ we show the probability distribution for finding a mode with a participation ratio $P$ for the modes with $P<P_{0}$ for $N$ $=48,000$ particles. With decreasing $T_{\mathrm{p}}$, the distribution becomes

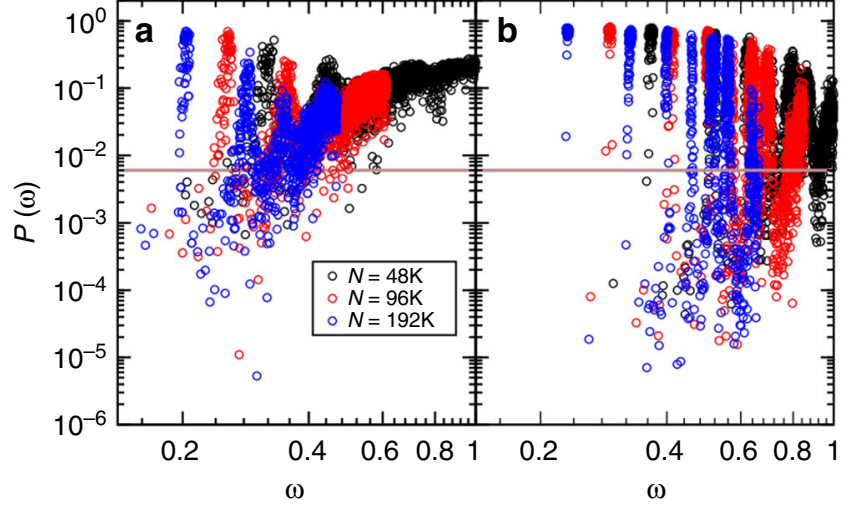

Fig. 2 Classification of modes. Participation ratio $P(\omega)$ as a function of frequencies $\omega$ combined from systems with $N=48,000,96,000$, and 192,000 at parent temperatures $T_{p}=0.200$ in (a) and $T_{p}=0.062$ in (b). The horizontal line marks the threshold $P_{0}=0.006$ between extended and quasi-localized modes

narrower and the peak position shifts to smaller $P$ values. We find that the average participation ratio decreases with decreasing $T_{\mathrm{p}}$, which is evident from Fig. 3c. This confirms that these modes become more localized with decreasing parent temperature, which had been observed for less stable glasses ${ }^{9,13,32}$. Since the density of states is a function of the structure of the quenched system, we conclude that subtle local structural changes occur for $T_{\mathrm{p}}$ below $T_{\mathrm{c}}$ that strongly affect soft vibrational motion in the quenched glass.

To visualize the increasing mode localization, we define a 'softness' 25 for particle $i$ as $A(i)=(1 / M) \sum_{l=1}^{M}\left|\mathbf{e}_{l, i}\right|$, where the sum is taken over the $M=40$ lowest frequency quasi-localized modes for one inherent structure (we have checked that our conclusions hold when we take other values of $M=5-40$ ). The softness quantifies the vibrational amplitude of low-frequency quasi-localized modes. In the snapshots of Fig. 4, particles are represented with a size proportional to $A(i)$ for (a) $T_{\mathrm{p}}=0.200$ and (b) $T_{\mathrm{p}}=0.062$. For the highest $T_{\mathrm{p}}$, clusters contributing to localized modes are relatively numerous, quite extended, and strongly coupled to their environment. At the lowest $T_{p}$, each cluster is localized around just a few particles, there are much fewer clusters, and they offer a stronger contrast with the 

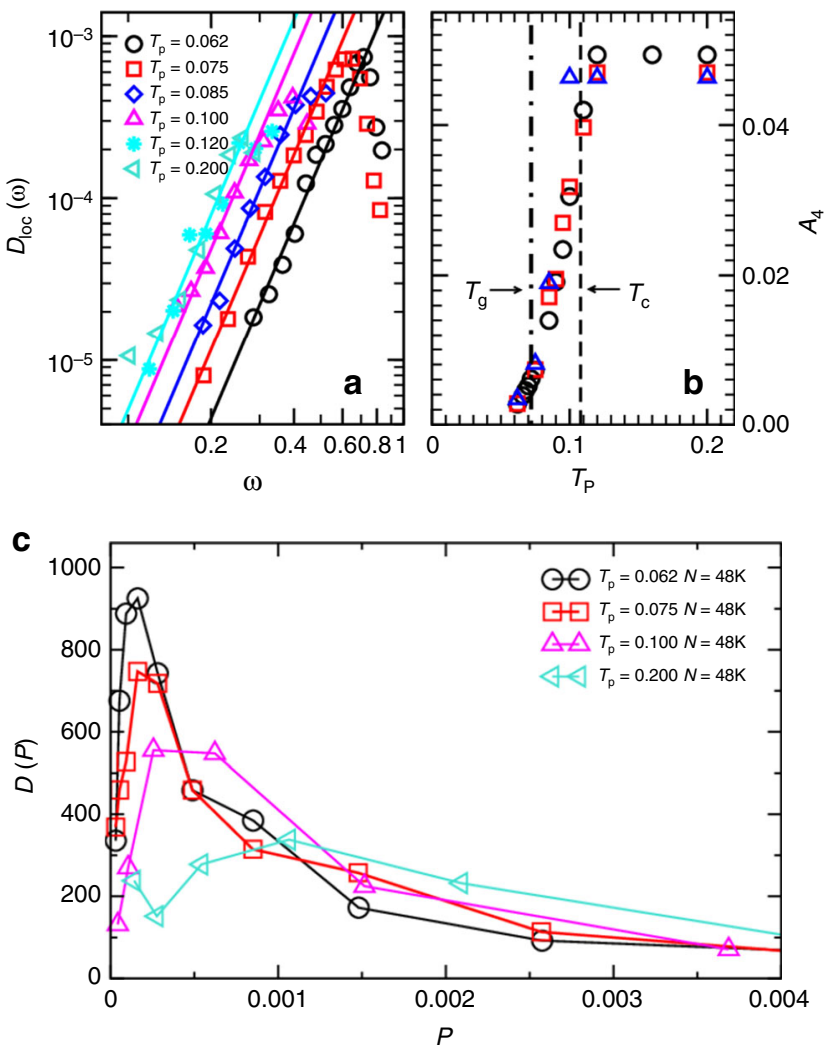

Fig. 3 Density of states and spatial localization of quasi-localized modes. a Density of states $D_{\text {loc }}(\omega)$ for quasi-localized modes for $N=48,000$, with fits to $D_{\text {loc }}=A_{4} \omega^{4}$. $\mathbf{b} T_{\mathrm{p}}$ dependence of $A_{4}$ for $N=48,000$ (circles), 96,000 (squares), and 192,000 (triangles), with the mode-coupling temperature $T_{\mathrm{c}}$ and the estimated experimental glass temperature $T_{\mathrm{g}}$ indicated. c Probability distribution of the participation ratio for quasilocalized modes in the frequency-range of the $\omega^{4}$ scaling for various $T_{\mathrm{p}}$

immobile background. To quantify these observations, we measured the probability distribution of $A(i)$ (Fig. 4c). These distributions show a power-law tail at large $A$ values, $P\left(A_{i}\right)=$ $\lambda\left(T_{\mathrm{p}}\right) A_{i}^{-\alpha}$ with $\alpha \approx 3.7$. At low $T_{\mathrm{p}}$ the tail is well separated from the core of the distribution at small $A$, and mobile particles with large $A$ are better defined. There is also a pronounced decay of the probability of finding large $A$ values at low $T_{\mathrm{p}}$ since $\lambda(0.2) / \lambda$ $(0.062) \approx 4.3$, which indicates a greater than four fold decrease in the number of soft particles with large vibrational amplitudes. The interpretation of quasi-localized modes as relevant glassy defects controlling mechanical and thermal properties of glasses is therefore more convincing for stable glasses than it is for conventional computer glasses.

Properties of extended modes. Next, we examine the density of states of extended modes, $D_{\text {ex }}(\omega)$, with a participation ratio greater than $P_{0}$. In Fig. $5 \mathrm{a}, \mathrm{b}$ we show the reduced density of states $D_{\text {ex }}(\omega) / \omega^{2}$ for two parent temperatures. For each temperature, the Debye level is reached at low enough $\omega$ and a boson peak is observed at larger frequencies. Using our localization criterion, we find that modes near the boson peak are not localized, but this does not imply that they have a phononic character. The boson peak narrows slightly with decreasing $T_{\mathrm{p}}$. The Debye level, the boson peak location, height, and width all change modestly as $T_{\mathrm{p}}$ is varied over the entire range studied. The changes observed in our study agree qualitatively with those found by Grigera et al. ${ }^{4}$.

In Fig. $5 c$ we examine scaling properties of the density of states of extended modes. We rescale $\omega$ by the boson peak frequency, a

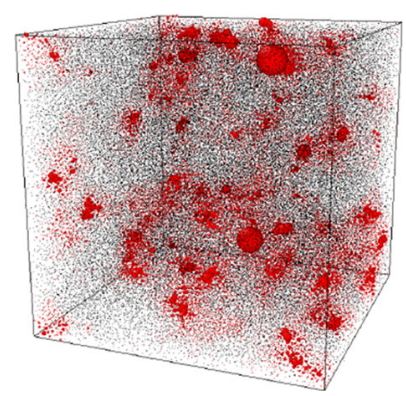

b

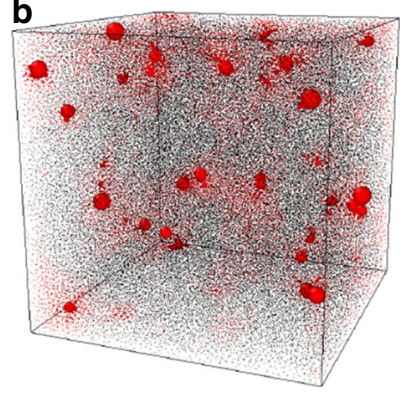

C

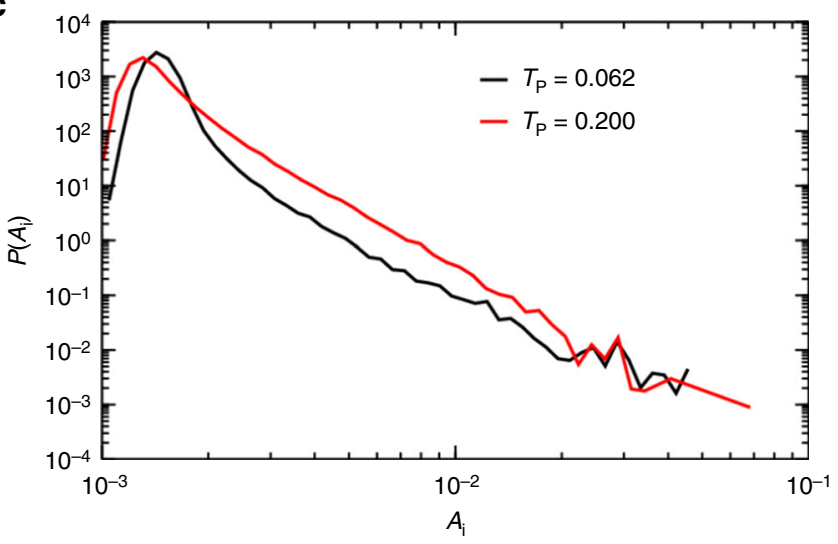

Fig. 4 Softness of quasi-localized modes. Snapshots obtained for $T_{p}=$ 0.200 (a) and $T_{p}=0.062$ (b) with $N=192,000$. The particles are shown with their radius given by the vibrational amplitude $A(i)$ calculated from the lowest quasi-localized modes. $\mathbf{c}$ The probability distribution of $A(i)$ for $T_{\mathrm{p}}=$ 0.200 and $T_{p}=0.062$. For lower parent temperatures there is a smaller fraction of the particles with larger $A(i)$, and thus the modes are more localized

$\omega_{\mathrm{BP}}$, and plot the rescaled density of states $D_{\mathrm{ex}} /\left(A_{\mathrm{D}} \omega^{2}\right)$. We observe an excellent collapse on the low-frequency side of the boson peak. This shows that in this frequency range the reduced frequency dependence has a universal shape, as reported before ${ }^{44}$. Second, the collapse also shows that the height of the boson peak correlates with the Debye level $A_{\mathrm{D}}$. These results agree with experiments on molecular glass formers ${ }^{45-47}$. However, some of the same experiments report that the boson peak position scales as the Debye frequency 45,47 , which is not consistent with our results. We also find that a scaling of $\omega_{\mathrm{BP}}$ with the bulk modulus suggested in ref. ${ }^{48}$ is inconsistent with our results. Note that we study the evolution of the boson peak as a function of the preparation temperature, while experiments sometimes examine the temperature evolution of the boson peak for a given glass preparation. We also note that a correlation between the boson peak and quasi-localized modes has been proposed by studying systems at different pressures around the unjamming transition ${ }^{49}$.

Since the boson peak occurs in a different frequency range than the $\omega^{4}$ scaling of $D_{\text {loc }}(\omega)$, it is not clear that there could be a relationship between the boson peak and the low-frequency quasi-localized modes. Simulations close to jamming suggest that $A_{4} \sim \omega_{\mathrm{BP}}^{4}{ }^{8}$, but we do not find that this relation holds with changing $T_{\mathrm{p}}$. An alternative possibility can be obtained from dimensional analysis, where a characteristic frequency for quasilocalized modes can be defined as $A_{4}^{-1 / 5} 32$. We find that $A_{4}^{-1 / 5} \sim$ $\omega_{\mathrm{BP}}$ for glasses with $T_{\mathrm{p}}<T_{\mathrm{c}}$ (Fig. 6), but this relation does not hold for glasses created with $T_{\mathrm{p}}>T_{\mathrm{c}}$. We note that $\omega_{\mathrm{BP}}$ is constant for $T_{\mathrm{p}}>T_{\mathrm{c}}$, see the inset to Fig. 6 , and only changes for $T_{\mathrm{p}}<T_{\mathrm{c}}$. Again we find that $T_{\mathrm{c}}$ marks a change in the behavior of $D(\omega)$. Given the relatively small changes in both $\omega_{\mathrm{BP}}$ and $A_{4}^{-1 / 5}$ over our 

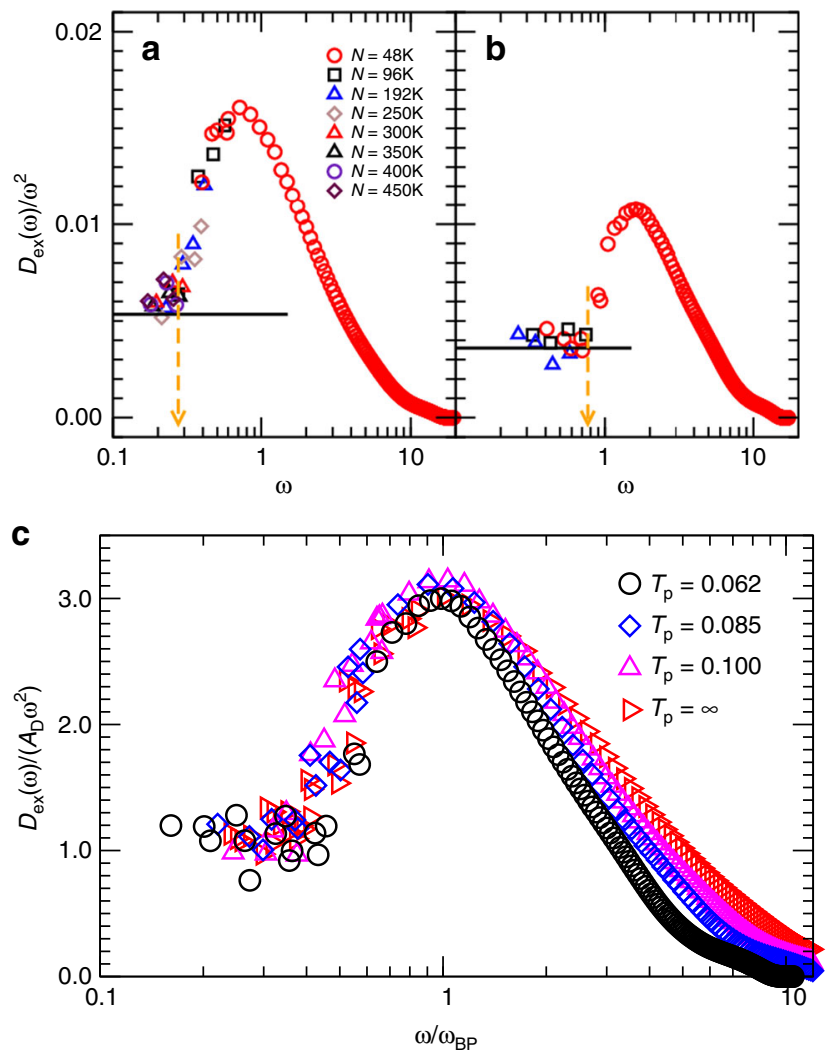

Fig. 5 Density of states for extended modes. Reduced density of states for extended modes, $D_{\text {ex }}(\omega) / \omega^{2}$, at parent temperatures $T_{\mathrm{p}}=\infty(\mathbf{a})$ and 0.062 (b), for systems with different sizes. The black line in each panel indicates the Debye level $A_{D}$ while the vertical arrow marks the frequency, where $D_{\text {ex }}(\omega) / \omega^{2}$ starts to deviate from $A_{D}$. c Rescaled version of the same data using the Debye level (vertical axis) and the boson peak frequency $\omega_{\mathrm{BP}}$ (horizontal axis)

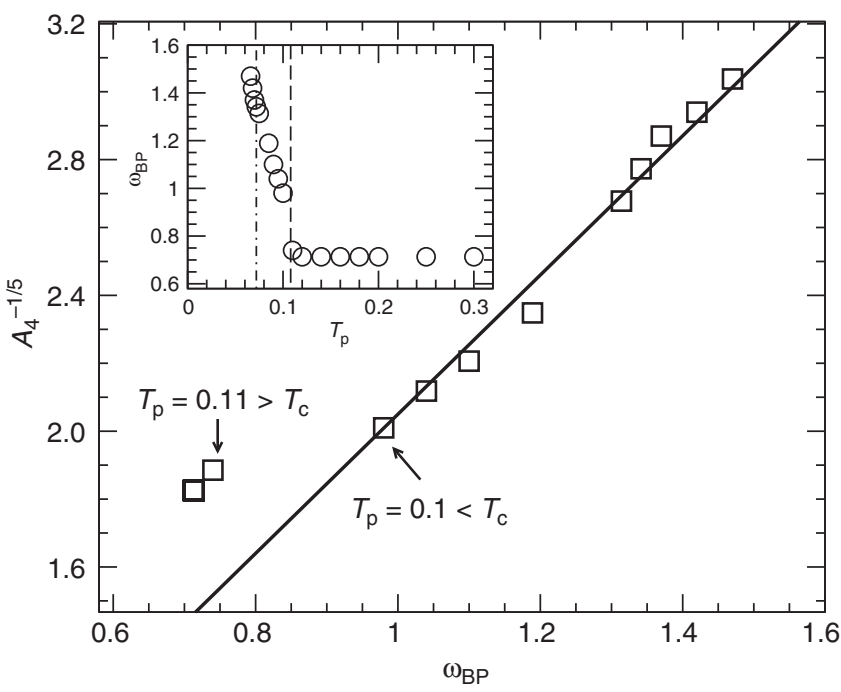

Fig. 6 Scaling of the boson peak frequency. The characteristic frequency $A_{4}^{-1 / 5}$ versus the boson peak position $\omega_{\mathrm{BP}}$. The line is a fit $A_{4}^{-1 / 5} \sim \omega_{\mathrm{BP}}$ for glasses whose $T_{\mathrm{p}}<T_{\mathrm{c}}$, which is the parent temperature range where we see an increase in $\omega_{\mathrm{BP}}$ with decreasing $T_{\mathrm{p}}$, see inset where the vertical dasheddotted, dashed lines mark the positions of $T_{g}$ and $T_{c}$, respectively entire range of parent temperatures studied, it is not clear that a power law is the proper relationship between these quantities and further work is needed to verify it.

\section{Discussion}

In summary, we report the first characterization of the vibrational density of states of computer glasses prepared over a range of glass stability that bridges the gap between ordinary simulations and experimental studies. At low-frequency extended and quasilocalized modes coexist, and both types of modes evolve differently when the glass stability is varied. We find a relatively mild temperature dependence of extended modes, with a strong correlation between the Debye level and the boson peak. By contrast, quasi-localized modes evolve more strongly when $T_{\mathrm{p}}$ decreases below the mode-coupling temperature, but their density of states is always described by $D_{\mathrm{loc}} \sim A_{4} \omega^{4}$. Unexpectedly, the temperature dependence of the prefactor $A_{4}\left(T_{\mathrm{p}}\right)$ is more interesting than the value of the quartic exponent, which is insensitive to the degree of annealing.

The increasing localization of the modes implies that subtle yet significant changes occur in the local structure of the glass that are not reflected in the pair correlation function, which is nearly identical for parent temperatures below $T_{\mathrm{c}}$. Since soft modes have been linked to irreversible relaxation ${ }^{24}$ and rearrangements under shear $^{25-28}$, it follows that the reduction of these soft modes can have significant implications for glassy dynamics. In turn this reduction indicates that there are fewer soft spots, which should increase the strength of the glass. This hypothesis is supported by the observation that the decrease in $D_{\text {loc }}(\omega)$ mirrors the increase of the shear modulus, and also correlates very well with the evolution of the ductility of the produced glasses ${ }^{38,50}$. Since we can now equilibrate amorphous systems at temperatures low enough so that they do not flow, another perspective would be to analyze the density of states at finite temperatures through the Fourier transform of the velocity autocorrelation function ${ }^{51}$, or by diagonalizing the covariance matrix of displacements ${ }^{52}$. Future studies should examine the difference between these procedures to provide insights into thermal anharmonicities of stable glasses, and more generally into their low-temperature transport properties.

\section{Methods}

Simulations. We simulate a polydisperse model glass former of sizes between $N=$ 48,000 and 450,000 particles with equal mass at a number density $\rho_{2}=1.0^{33}$. The interaction between two particles $i$ and $j$ is given by $V\left(r_{i j}\right)=\left(\frac{\sigma_{i j}}{r_{i j}}\right)^{12}+v\left(r_{i j}\right)$ when their separation $r_{i j} \leq r_{i j}^{c}=1.25 \sigma_{i j}$ and zero otherwise. We use

$v\left(r_{i j}\right)=c_{0}+c_{2}\left(\frac{r_{i j}}{\sigma_{i j}}\right)^{2}+c_{4}\left(\frac{r_{i j}}{\sigma_{i j}}\right)^{4}$, where the coefficients $c_{0}, c_{2}$, and $c_{4}$ ensure the continuity of $V\left(r_{i j}\right)$ up to the second derivative at the cutoff $r_{i j}^{c}$. The probability of particle diameters $\sigma$ is $P(\sigma)=A / \sigma^{3}$, where $\sigma \in[0.73,1.63]$ and we use a non-additive mixing rule, $\sigma_{i j}=\frac{\sigma_{i}+\sigma_{j}}{2}\left(1-0.2\left|\sigma_{i}-\sigma_{j}\right|\right)$. For $N \leq 192,000$ we use the swap Monte Carlo algorithm to prepare independent equilibrated configurations at parent temperatures $T_{\mathrm{p}}$ ranging from above the onset temperature of slow dynamics $\left(T_{\mathrm{o}} \approx\right.$ 0.200 ) down to $T_{\mathrm{p}}=0.062$, which is about $60 \%$ of the mode-coupling temperature $\left(T_{\mathrm{c}} \approx 0.108\right)$, and is lower than the estimated experimental glass temperature $\left(T_{\mathrm{g}} \approx\right.$ $0.072)^{33}$. In addition, we also use a very high parent temperature, which we refer to as $T_{\mathrm{p}}=\infty$. Due to very long equilibration times for systems of $N>192,000$ particles we only study systems with $N>192,000$ for $T_{\mathrm{p}}=\infty$.

Density of states calculation. Following equilibration at a temperature $T_{\mathrm{p}}$, zerotemperature glasses are produced by instantaneously quenching equilibrium configurations to their inherent structures using the Fast Inertia Relaxation Engine algorithm ${ }^{53}$. We then calculate the modes by diagonalizing the Hessian matrix using Intel Math Kernel Library (https://software.intel.com/en-us/mkl/) and ARPACK (http://www.caam.rice.edu/software/ARPACK/). We calculate all the normal modes for the 48,000 particle systems, but only the low-frequency part of the spectrum in systems with $N>48,000$. We characterize the modes through the density of states $D(\omega)=\frac{1}{3 N-3} \sum_{l=1}^{3 N-3} \delta\left(\omega-\omega_{l}\right)$ and the participation ratio 
$P\left(\omega_{l}\right)=\frac{\left.\left(\sum_{i=1}^{N} \mid \mathbf{e}_{l, i}\right)^{2}\right)^{2}}{N \sum_{i=1}^{N}\left|\mathbf{e}_{l, i}\right|^{4}}$, where $\boldsymbol{e}_{l, i}$ is the polarization vector of particle $i$ in mode $l$ with frequency $\omega_{l}$. For a mode localized to one particle $P(\omega)=N^{-1}$, and for an ideal plane wave $P(\omega)=2 / 3$. The phonon modes occur at discrete frequencies, and care has to be taken in the binning procedure to calculate the density of states of extended modes, $D_{\mathrm{ex}}(\omega)$. To perform this calculation, we determine the phonon frequencies from the peak positions of the participation ratio versus frequency, and tune the bin size to smooth $D_{\text {ex }}(\omega)$.To obtain the shear modulus $G$ and the bulk modulus $B$ we use the method described in ref. ${ }^{54}$.

\section{Data availability}

All data will be available from the authors upon request.

Received: 28 August 2018 Accepted: 4 December 2018

Published online: 03 January 2019

\section{References}

1. Zeller, R. C. \& Pohl, R. O. Thermal conductivity and specific heat of noncrystalline solids. Phys. Rev. B 4, 2029-2041 (1971).

2. Kittel, C. Introduction to Solid State Physics. 7th edn (Wiley, New York, 1996).

3. Nakayama, T. Boson peak and terahertz frequency dynamics of vitreous silica. Rep. Prog. Phys. 65, 1195-1242 (2002).

4. Grigera, T. S., Martin-Mayor, V., Parisi, G. \& Verrocchio, P. Phonon interpretation of the boson peak in supercooled liquids. Nature 422, 289-292 (2003).

5. Inoue, K. et al. Low-energy excitations in amorphous polymers. J. Chem. Phys. 95, 5332-5340 (1991).

6. Buchenau, U., Wischnewski, A., Ohl, M. \& Fabiani, E. Neutron scattering evidence on the nature of the boson peak. J. Phys. Condens. Matter 19, 205106 (2007).

7. Lerner, E., Düring, G. \& Bouchbinder, E. Statistics and properties of lowfrequency vibrational modes in structural glasses. Phys. Rev. Lett. 117, 035501 (2016).

8. Mizuno, H., Shiba, H. \& Ikeda, A. Continuum limit of the vibrational properties of amorphous solids. Proc. Natl Acad. Sci. USA 114, E9767-E9774 (2017).

9. Lerner, E. \& Bouchbinder, E. Effect of instantaneous and continuous quenches on the density of vibrational modes in model glasses. Phys. Rev. E 96, 020104 (R) (2017).

10. Shimada, M., Mizuno, H. \& Ikeda, A. Anomalous vibrational properties in the continuum limit of glasses. Phys. Rev. E 97, 022609 (2018).

11. Kapteijns, G., Bouchbinder, E. \& Lerner, E. Universal nonphononic density of states in 2D, 3D, and 4D glasses. Phys. Rev. Lett. 121, 055501 (2018).

12. Angelani, L., Paoluzzi, M., Parisi, G. \& Ruocco, G. Probing the non-Debye low-frequency excitations in glasses through random pinning. Proc. Natl Acad. Sci. USA 115, 8700-8704 (2018).

13. Lerner, E. \& Bouchbinder, E. Frustration-induced internal stresses are responsible for quasilocalized modes in structural glasses. Phys. Rev. E 97, 032140 (2018).

14. Buchenau, U., Galperin, Yu. M., Gurevich, V. L. \& Schober, H. R. Anharmonic potentials and vibrational localization in glasses. Phys. Rev. B 43, 5309 (1991). -5045 .

15. Buchenau, U. et al. Interaction of soft modes and sound waves in glasses. Phys. Rev. B 46, 2798-2808 (1992).

16. Schober, H. R. \& Oligschleger, C. Low-frequency vibrations in a model glass. Phys. Rev. B 53, 11469-11480 (1996).

17. Gurevich, V. L., Parshin, D. A. \& Schober, H. R. Anharmonicity, vibrational instability, and the boson peak in glasses. Phys. Rev. B 67, 094203 (2003).

18. Schirmacher, W., Ruocco, G. \& Scopigno, T. Acoustic attenuation in glasses and its relation with the boson peak. Phys. Rev. Lett. 98, 025501 (2007).

19. Benetti, F. P. C., Parisi, G., Pietracaprina, F. \& Sicuro, G. Mean-field model for the density of states of jammed soft spheres. Phys. Rev. E 97, 062157 (2018).

20. Stanifer, E., Morse, P. K., Middleton, A. A. \& Manning, M. L. A simple random matrix model for the vibrational spectrum of jammed packings. Preprint at http://arXiv.org/abs/1804.04074 (2018).

21. DeGiuli, E., Laversanne-Finot, A., Düring, G., Lerner, E. \& Wyart, M. Effects of coordination and pressure on sound attenuation, boson peak and elasticity in amorphous solids. Soft Matter 10, 5628-5644 (2014).

22. Franz, S., Parisi, G., Urbani, P. \& Zamponi, F. Universal spectrum of normal modes in low-temperature glasses. Proc. Natl Acad. Sci. USA 112, 14539-14544 (2015).

23. Charbonneau, P., Corwin, E. I., Parisi, G., Poncet, A. \& Zamponi, F. Universal non-Debye scaling in the density of states of amorphous solids. Phys. Rev. Lett. 117, 045503 (2016).
24. Widmer-Cooper, A., Perry, H., Harrowell, P. \& Reichman, D. R. Irreversible reorganization in a supercooled liquid originates from localized soft modes. Nat. Phys. 4, 711-715 (2008).

25. Schoenholz, S. S., Liu, A. J., Riggleman, R. A. \& Rottler, J. Understanding plastic deformation in thermal glasses from single-soft-spot dynamics. Phys Rev. X 4, 031014 (2014).

26. Chen, K. et al. Measurement of correlations between low-frequency vibrational modes and particle rearrangements in quasi-two-dimensional colloidal glasses. Phys. Rev. Lett. 107, 108301 (2011).

27. Manning, M. L. \& Liu, A. J. Vibrational modes identify soft spots in a sheared disordered packing. Phys. Rev. Lett. 107, 108302 (2011).

28. Zylberg, J., Lerner, E., Bar-Sinai, Y. \& Bouchbinder, E. Local thermal energy as a structural indicator in glasses. Proc. Natl Acad. Sci. USA 114, 7289-7294 (2017).

29. Phillips, W. A. Tunneling states in amorphous solids. J. Low Temp. Phys. 7, 351-360 (1972).

30. Anderson, P. W., Halperin, B. I. \& Varma, C. M. Anomalous low-temperature thermal properties of glasses and spin glasses. Philos. Mag. 25, 1-9 (1972)

31. Berthier, L. \& Biroli, G. Theoretical perspective on the glass transition and amorphous materials. Rev. Mod. Phys. 83, 587-645 (2011).

32. Lerner, E. \& Bouchbinder, E. A characteristic energy scale in glasses. J. Chem. Phys. 148, 214502 (2018).

33. Ninarello, A., Berthier, L. \& Coslovich, D. Models and algorithms for the next generation of glass transition studies. Phys. Rev. X 7, 021039 (2017).

34. Berthier, L., Coslovich, D., Ninarello, A. \& Ozawa, M. Equilibrium sampling of hard spheres up to the jamming density and beyond. Phys. Rev. Lett. 116, 238002 (2016).

35. Fullerton, C. \& Berthier, L. Density controls the kinetic stability of ultrastable glasses. Europhys. Lett. 119, 36003 (2017).

36. Berthier, L. et al. Configurational entropy measurements in extremely supercooled liquids that break the glass ceiling. Proc. Natl Acad. Sci. USA 114, 11356-11361 (2017).

37. Scalliet, C., Berthier, L. \& Zamponi, F. Absence of marginal stability in a structural glass. Phys. Rev. Lett. 119, 205501 (2017).

38. Ozawa, M., Berthier, L., Biroli, G., Rosso, A. \& Tarjus, G. Random critical point separates brittle and ductile yielding transitions in amorphous materials. Proc. Natl Acad. Sci. USA 115, 6656-6661 (2018).

39. Pérez-Castañeda, T., Rodrguez-Tinoco, C., Rodrguez-Viejo, J. \& Ramos, M. A. Suppression of tunneling two-level systems in ultrastable glasses of indomethacin. Proc. Natl Acad. Sci. USA 111, 11275-11280 (2014).

40. Liu, X., Queen, D. R., Metcalf, T. H. J., Karel, E. \& Hellman, F. Hydrogen-free amorphous silicon with no tunneling states. Phys. Rev. Lett. 113, 025503 (2014).

41. Ramos, M. A. \& Pérez-Castañeda, T. Do tunneling states and boson peak persist or disappear in extremely stabilized glasses? J. Low Temp. Phys. 41, 412-418 (2015).

42. Helfferich, J., Lyubimov, I., Reid, D. \& de Pablo, J. J. Inherent structure energy is a good indicator of molecular mobility in glasses. Soft Matter, 12, 5898-5904 (2016).

43. Sastry, S., Debenedetti, P. G. \& Stillinger, F. H. Signatures of distinct dynamical regimes in the energy landscape of a glass-forming liquid. Nature 393, 554-557 (1998).

44. Shintani, H. \& Tanaka, H. Universal link between the boson peak and transverse phonons in glass. Nat. Mater. 7, 870-877 (2008).

45. Monaco, A. et al. Density of vibrational states of a hyperquenched glass. Phys. Rev. Lett. 96, 205502 (2006)

46. Baldi, G. et al. Connection between boson peak and elastic properties in silicate glasses. Phys. Rev. Lett. 102, 195502 (2009).

47. Monaco, A. et al. Effect of densification on the density of vibrational states of glasses. Phys. Rev. Lett. 97, 135501 (2006).

48. Rufflé, B. et al. Scaling the temperature-dependent boson peak of vitreous silica with the high-frequency bulk modulus derived from Brillouin scattering data. Phys. Rev. Lett. 104, 067402 (2010).

49. Shimada, M., Mizuno, H., Wyart, M. \& Ikeda, A. Spatial structure of quasilocalized vibrations in nearly jammed amorphous solids. Preprint at http:// arXiv.org/abs/1804.08865 (2018).

50. Ketkaew, J. et al. Mechanical glass transition revealed by the fracture toughness of metallic glasses. Nat. Commun. 9, 3271 (2018).

51. Keyes, T. Instantaneous normal mode approach to liquid state dynamics. $J$. Phys. Chem. A 101, 2921-2930 (1997).

52. Henkes, S., Brito, C. \& Dauchot, O. Extracting vibrational modes from fluctuations: a pedagogical discussion. Soft Matter 8, 6092-6109 (2012).

53. Bitzek, E., Koskinen, P., Gähler, F., Moseler, M. \& Gumbsch, P. Structural relaxation made simple. Phys. Rev. Lett. 97, 170201 (2006).

54. O’Hern, C. S., Silbert, L. E., Liu, A. J. \& Nagel, S. R. Jamming at zero temperature and zero applied stress: The epitome of disorder. Phys. Rev. E 68 011306 (2003). 


\section{Acknowledgements}

We thank J.-L. Barrat for discussions. L.W., E.F., and G.S. acknowledge funding from NSF DMR-1608086. This work was also supported by a grant from the Simons foundation (No. 454933 L.B.). L.W. and P.G. acknowledge support from the National Natural Science Foundation of China (No. 51571011), the MOST 973 Program (No.

2015CB856800), and the NSAF joint program (No. U1530401). We acknowledge the computational support from Beijing Computational Science Research Center.

\section{Author contributions}

L.B., G.S., and E.F. designed the project. L.W. and A.N. performed numerical simulations. L.W., A.N., L.B., P.G., G.S., and E.F. contributed to the analysis of data. L.W., L.B., G.S., and E.F. wrote the paper.

\section{Additional information}

Competing interests: The authors declare no competing interests.

Reprints and permission information is available online at http://npg.nature.com/ reprintsandpermissions/
Journal peer review information: Nature Communications thanks Miguel Ramos and the other anonymous reviewer(s) for their contribution to the peer review of this work

Publisher's note: Springer Nature remains neutral with regard to jurisdictional claims in published maps and institutional affiliations.

(c) Open Access This article is licensed under a Creative Commons Attribution 4.0 International License, which permits use, sharing, adaptation, distribution and reproduction in any medium or format, as long as you give appropriate credit to the original author(s) and the source, provide a link to the Creative Commons license, and indicate if changes were made. The images or other third party material in this article are included in the article's Creative Commons license, unless indicated otherwise in a credit line to the material. If material is not included in the article's Creative Commons license and your intended use is not permitted by statutory regulation or exceeds the permitted use, you will need to obtain permission directly from the copyright holder. To view a copy of this license, visit http://creativecommons.org/licenses/by/4.0/.

(C) The Author(s) 2019 